\title{
Changes in Phytotoxicity during High-rate Anaerobic Digestion and Sequential Aerobic Composting for Sewage Sludge Stabilization
}

\author{
Yanfei Tang ${ }^{1}$, Xiaowei $\mathrm{Li}^{1,2}$, Bin Dong ${ }^{1}$, Lingling Dai ${ }^{1}$, Junjie Huang ${ }^{1}$, Yanhong \\ $\mathrm{Wei}^{1}$, and Xiaohu Dai ${ }^{1, a}$ \\ ${ }^{1}$ College of Environment Science and Engineering, Tongji University, Shanghai 200092, China \\ ${ }^{2}$ Department of environmental science and engineering, College of environmental and chemical \\ engineering, Shanghai University, Shanghai 200092, China \\ ${ }^{a}$ Corresponding author: daixiaohu@tongji.edu.cn
}

Keywords: Sludge Phytotoxicity; Stabilization treatment; Humification; Land application.

Abstract. Seed germination and germinated seedlings growth in sewage sludge were analyzed using acute and subchronic phytotoxicity assays, in order to explore the changes in sludge phytotoxicity during the combination of high-rate anaerobic digestion and aerobic composting. Results showed that compared with the raw sludge (RS), the seed germination index (GI) and shoot length of germinated seedlings of anaerobic digested sludge (ADS) reduced after anaerobic digestion. It implied the high-rate anaerobic digestion (AD) caused an increase of the sludge phytotoxicity. After 14-day Sequential aerobic composting, shoot length, fresh and dry shoot biomass of the seedlings in the aerobic compost sludge (ACS) significantly enhanced, compared with that of the ADS. As the composting lasted from $14 \mathrm{~d}$ to $60 \mathrm{~d}$, there was not an evident increasing trend in seed germination and germinated seedlings growth of the sludge. Additionally, humification indexes significantly correlated with GI, and the negative slope at ACS 60d was in accordance with the decreasing trend of plant growth. The findings indicate the 14-day sequential aerobic composting was necessary and feasible for the complement of high-rate anaerobic digestion of sewage sludge in consideration of the phytotoxicity reduction.

\section{Introduction}

Owing to rapid urbanization, huge amount of sewage sludge produced in China is becoming a serious environmental challenge. It was estimated at 35 million tons in 2015 and is projected to reach 60 to 90 million tons by 2020 [1]. The anaerobic digested and compost sludge were considered to have a high potential fertilizer value by their contents of $\mathrm{N}, \mathrm{P}, \mathrm{K}$ and micronutrients [2]. Thus, land application is an important disposal route and now it's the trend not only in European countries but also in China $[3,4]$. However, parameters as electrical conductivity, ammonium nitrogen and the behavior of heavy metals in sludge are among the factors of phytotoxicity which limit sludge application in soil $[2,5,7]$. Therefore, it is needed to evaluate whether the major stabilization treatments including anaerobic digestion and aerobic composting can reduce phytotoxicity effectively before application to soil.

Nowadays, evaluating ecological risk i.e. estimating potential consequence under a particular human action towards environment can help taking decisions and predicting risk in the future [8]. Furthermore, the most evident effects of toxic compounds including the inhibition of seed germination and root growth were found $[9,12]$, and the phytotoxicity assays are becoming more and more common in ecotoxicology due to the easiness for handling, low comsumption and the better sensitiveness under environmental stress than other living organisms [10,11]. However, the assay using sludge extracts do not always reflect the level of toxicity present in soil, suggested the importance of testing directly on soil matrices for standardising phytotoxicity $[9,12]$. Up to now, few papers discussed changes in phytotoxicity during the sludge stabilization treatment of high-rate anaerobic digestion and aerobic composting using acute germination assay and subchronic plant tests.

It was discovered ammonium and organic matter stability were the most influential determinants but not pollutant burden for phytotoxicity $[8,18]$, because after most of the easily available organic matter had been decomposed then the humification started and influenced phytotoxicity [13]. Additionally, the humification reactions are related to the degradations of carbohydrates, proteins, 
amino acids, lipids, and lignins into simpler compounds [14]. The objectives of the study were: 1) to explore the relation of humicfication indices and GI from high-rate anaerobic digestion and aerobic compost in three plants; 2) to investigate the 48-hour seed germination and 14-day germinated seedlings growth using sludge and peat (dry weight $1: 1$ ).

\section{Materials and Methods}

Materials. The substrates properties were shown in Table1. Dewatered sewage sludge was obtained from the dumping site of Quyang WWTP, Shanghai, China. The moisture content of the sludge was about $75-85 \%$. The ADS was collected from a mesothermal high-rate anaerobic digestion reactor using the dewatered sludge as the feed, which was operated for more than 48 days. Then ADS was dewatered to $75-85 \%$ of the moisture content. The dewatered sludge was used as the substrate of the aerobic composting. The aerobic composting (AC) took place in a $40 \mathrm{~L}$ reactor with an aeration rate of 200-300 L h$~^{-1}$. Because of the restriction of sewage sludge in agriculture in China, this study selected plant species among the most sensitive plant species recommended by the OECD (1984) except vegetables. They were morning glory (Ipomoea hederacea L.), cornflower (Centaurea cyanus L.) and sunflower (Helianthus annuus L.), respectively called MG, CF and SF in this study.

\section{Method}

Physico-chemical parameters. Samples of the digestate were taken once every 8 days and analyzed for $\mathrm{pH}, \mathrm{EC}$ and VS. Total extractable carbon (TEC) obtained by shaking $2 \mathrm{~g}$ of freeze-dried sludge with $100 \mathrm{ml}$ of the solution of $0.1 \mathrm{M} \mathrm{Na}_{4} \mathrm{P}_{2} \mathrm{O}_{7}$ and $0.1 \mathrm{M} \mathrm{NaOH}$ for $48 \mathrm{~h}$ at $338 \mathrm{~K}$, and $25 \mathrm{ml}$ of the supernatant solution was acidified to $\mathrm{pH} \sim 1$ with $50 \% \mathrm{H}_{2} \mathrm{SO}_{4}$, the supernatant solution was fulvic acid-like fraction (FAL). Total organic carbon (TOC), the organic carbon in FAL fraction (FAL), TEC and the total extractable nitrogen (TEN) were measured by a TOC-VCPN analyzer (Shimadzu, Japan). The humification indices were calculated using the following expressions (Rolette et al., 1985):

Degree of polymerization $($ DP $)=$ HAC/FAC

Percentage of humic acids $(\mathrm{PHA})=\mathrm{HAC} / \mathrm{TEC}$

Acute seed germination tests. According to Chinese GB/T 23486-2009, germination (\%) and shoot length were measured and used as phytotoxicity endpoints. Sludge extracts were prepared by shaking $8 \mathrm{~g}$ about $85 \%$ moisture content of sludge with $24 \mathrm{ml}$ of distilled water for an hour in a speed of $160 \mathrm{r} / \mathrm{min}$. The suspension was then centrifuged before being introduced into a polyethylene tube and kept at $4{ }^{\circ} \mathrm{C}$. 20 seeds were placed on one layer of filter paper in $7.5 \mathrm{~cm}$ Petri dishes and $5 \mathrm{ml}$ of each sludge extract was added. Distilled water was used as a blank control. For each treatment and control group, the number of replicates is five. All the Petri dishes were then incubated in a growth chamber at $25{ }^{\circ} \mathrm{C}$ for 48 hours without photoperiod. GI was calculated as follows, in which GS, GB: germination $(\%)$ of the sludge and the control respectively, and LS, LB: average valid root length of seeds for the sludge and the control:

$$
\mathrm{GI}=100 \% \times \frac{G S \times \mathrm{LS}}{G B \times \mathrm{LB}}
$$

Subchronic germinated seedlings growth tests. The tests were performed in according with the procedures of the OECD Guideline 208A. Germination(\%), shoot length, fresh biomass and dry biomass were measured and used as phytotoxicity endpoints. Controlled substrate moisture at $60 \%$, and mix the peat with sludge as dry weight 1:1 then put into plastic pots. 20 seeds of each species were sown uniformly in each pot to a depth of approximately $3 \mathrm{~cm}$ then incubated in a growth chamber at $21^{\circ} \mathrm{C}, 16 / 8 \mathrm{~h}$ (light/dark) cycles. 


\section{Results and Discussion}

Physic and chemical characterization of RS, ADS and ACS. The results again confirmed the humification was continuous after mineralization [13]. The value of TOC, VS, TEC and FAC (Table 1) decreased in $\mathrm{AD}$ and $\mathrm{AC}$ mainly caused by the biodegradation. Meanwhile, the EC increased during $\mathrm{AD}$ and $\mathrm{AC}$ respectively. And $\mathrm{pH}$ increased from 7.49 of $\mathrm{RS}$ to 9.37 of $\mathrm{ADS} 40 \mathrm{~d}$ and decreased from ADS $48 \mathrm{~d}$ to 7.89 of ACS. The low values of $\mathrm{EC}$ and $\mathrm{pH}$ in $\mathrm{AC}$ were probably because the ammonia released. The value of HAC and TEN declined at first and then increased, ranged from $69.73 \mathrm{mg} / \mathrm{g}$ to $18.49 \mathrm{mg} / \mathrm{g}$ and from $51.8 \mathrm{mg} / \mathrm{g}$ to $14.3 \mathrm{mg} / \mathrm{g}$ respectively.

Table 1. Physic and chemical properties of different stages sludge substrate.

\begin{tabular}{lllllllll}
\hline & $\begin{array}{l}\text { TOC } \\
(\mathbf{m g} / \mathbf{g})\end{array}$ & $\begin{array}{l}\text { VS } \\
(\boldsymbol{\%})\end{array}$ & $\mathbf{p H}$ & $\begin{array}{l}\text { EC } \\
(\mathbf{m s} / \mathbf{c m})\end{array}$ & $\begin{array}{l}\text { TEC } \\
(\mathbf{m g} / \mathbf{g})\end{array}$ & $\begin{array}{l}\text { FAC } \\
(\mathbf{m g} / \mathbf{g})\end{array}$ & $\begin{array}{l}\text { HAC } \\
(\mathbf{m g} / \mathbf{g})\end{array}$ & $\begin{array}{l}\text { TEN } \\
(\mathbf{m g} / \mathbf{g})\end{array}$ \\
\hline RS & $339.8 \pm 21.7$ & $63.10 \pm 0.00$ & 7.49 & 0.62 & $156.1 \pm 9.6$ & $86.3 \pm 12.2$ & $69.73 \pm 21.8$ & $51.8 \pm 1.9$ \\
ADS 8d & $220.2 \pm 6.8$ & $47.97 \pm 0.00$ & 8.71 & 3.07 & $78.2 \pm 2.9$ & $40.5 \pm 8.0$ & $37.77 \pm 10.9$ & $22.6 \pm 0.5$ \\
ADS 16d & $226.5 \pm 5.6$ & $44.34 \pm 0.83$ & 8.84 & 3.20 & $82.7 \pm 1.1$ & $41.3 \pm 13.0$ & $41.43 \pm 14.1$ & $23.6 \pm 0.4$ \\
ADS 24d & $221.5 \pm 19.7$ & $43.52 \pm 0.62$ & 8.88 & 3.41 & $71.7 \pm 0.7$ & $39.6 \pm 25.5$ & $32.10 \pm 26.2$ & $20.7 \pm 1.0$ \\
ADS 32d & $203.9 \pm 10.0$ & $40.03 \pm 0.00$ & 9.34 & 2.85 & $68.3 \pm 0.3$ & $38.6 \pm 0.0$ & $29.69 \pm 0.3$ & $21.9 \pm 0.2$ \\
ADS 40d & $198.9 \pm 40.0$ & $41.89 \pm 1.16$ & 9.37 & 2.60 & $57.3 \pm 0.6$ & $38.8 \pm 45.5$ & $18.49 \pm 46.1$ & $19.6 \pm 0.9$ \\
ADS 48d & $196.2 \pm 33.2$ & $39.50 \pm 0.17$ & 9.13 & 3.73 & $59.2 \pm 10.8$ & $33.3 \pm 0.0$ & $25.89 \pm 10.8$ & $14.3 \pm 3.5$ \\
ACS 10d & $202.5 \pm 3.0$ & $50.73 \pm 2.31$ & 9.01 & 2.20 & $46.3 \pm 9.9$ & $27.0 \pm 5.7$ & $19.35 \pm 15.6$ & $17.6 \pm 0.1$ \\
ACS 14d & $202.2 \pm 17.6$ & $51.38 \pm 0.79$ & 8.37 & 2.37 & $46.4 \pm 1.7$ & $25.1 \pm 0.79$ & $21.39 \pm 2.49$ & $17.9 \pm 2.2$ \\
ACS 60d & $195.9 \pm 23.6$ & $44.14 \pm 0.47$ & 7.89 & 2.43 & $50.1 \pm 4.5$ & $22.05 \pm 1.4$ & $28.05 \pm 5.9$ & $22.3 \pm 2.3$ \\
\hline
\end{tabular}

Acute seed germination tests. A significant inhibition on the germination of three seeds was detected in the extracts of RS, ADS 32d and ADS 48d when compared to the Control (Fig.1). The ADS was toxic since the GI of ADS $32 \mathrm{~d}$ and ADS 48d were below 25\%, even both in CF and ADS 32d in MG were 0\%. However, a high reduction of phytotoxicity in ACS 14d suggested by the GI of 74\% on MG and 104\% on SF was observed though the incomplete stabilization and the plant sensitiveness may cause GI of ACS 14d in CF was 19\%. On the other hand, this result showed the digested sludge lost phytotoxicity with 14-day aerobic composting, thus stimulating seed germination and plant growth. Besides, high values of GI ranged from $117 \%$ to $341 \%$ were found after 60-day composting. As for average root length, it's found ADS would be more toxic with longer digestion, because ADS 48d had shorter root length than ADS 32d in MG and SF, and both were zero in CF. Though ACS 60d had the highest GI, its average root length was less than ADS14d in SF, possibly caused by higher EC value of ACS 60d i.e. high salinity [6]. Whereas, the heterogeneity of ADS may lead that ADS 32d had longer root length than RS in MG.

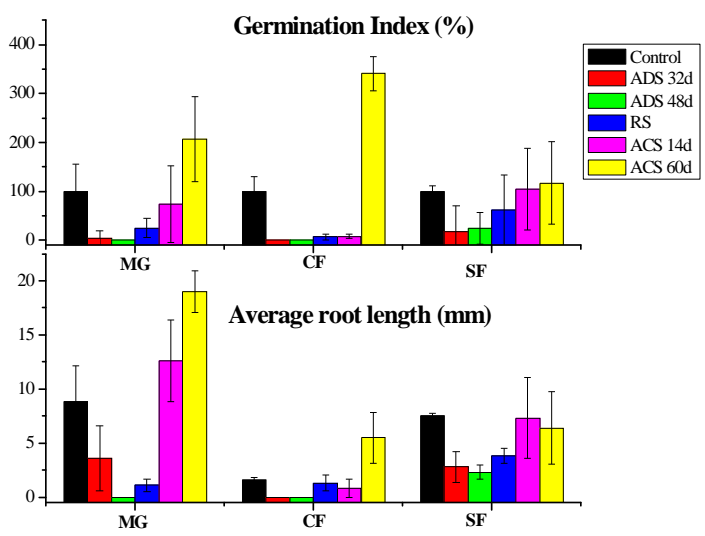

Fig.1. GI and root length of in 6 sludge extracts.

Subchronic germinated seedlings growth tests. It was evident $\mathrm{AD}$ was toxic and associated to an extremely high environmental risk [6], but AC was an effective treatment to reduce phytotoxicity [8]. To be effectively, seedlings growth tests focused on ACS and 6 samples in which were chosen, and ADS 48d and RS were for contrast. As showed in Fig.2, the sensitiveness of three plants on seedling 
emergence tested to the same sludge were different, especially the emergence of SF and CF in Control took place 1-2 days after sowing, but of MG was delayed and used 4 days. However, the diversities helped to simulate the real. The results of the 14-day germination sequence of ADS and RS were consistent with the seed germination tests. In ACS14d and ACS 60d, the emergence of SF, CF and MG took place almost simultaneously and quickly reached the maximum germination. But in ACS 2d, ACS 5d and ACS 10d, there was no significant trend of three plants. The ACS 10d had the highest germination in SF, but it had the lowest in CF. And the fluctuation also occurred in ACS 2d and ACS 5d. It could be explained 2-day, 5-day and 10-day compost were not stabilized enough until the composting lasted 14 days. In addition, the results of germination sequence and final germination (Table 2) in 14-day and 60-day composting were similar, which concluded it is not necessarily to compost too long prior to sludge reuse in soils. With different sizes of three seeds themselves, there were distinctions in fresh and dry shoot biomass (Fig.3). Compared to ACS14d, the decreases of three plants were found in ACS 60d of SF, also a little decrease was found in MG, which was agreed with the trend showed in root length of the 48-hour assays and shoot length (Table 2) of 14-day plants tests. To explain the trend in a new perspective, detail description is in the next section.

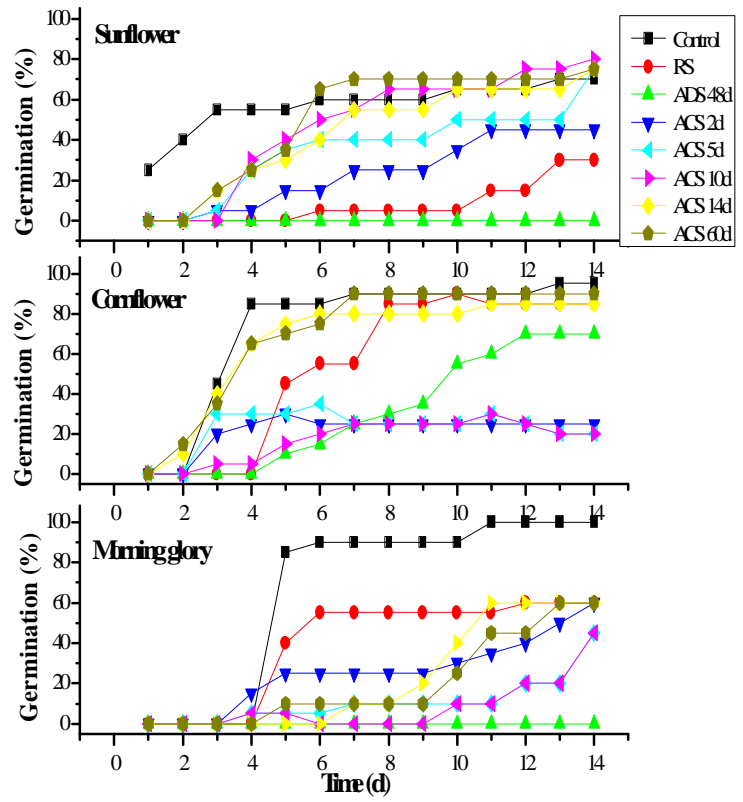

Fig. 2. Germination sequence in 8 sludges.

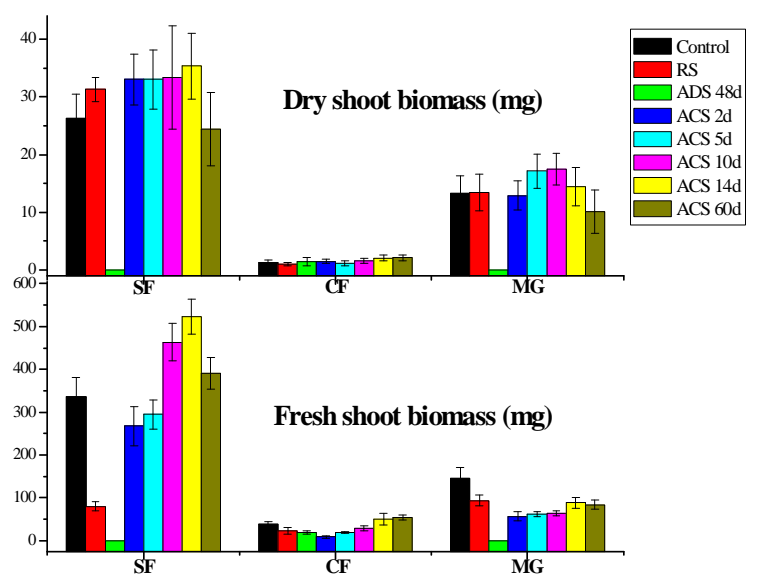

Fig.3. Fresh and dry shoot biomass in 8 sludges

Table 2. Comparison of the seed germination, shoot length of $14 \mathrm{~d}$ seeding between 8 kinds of sludge.

\begin{tabular}{llllllllll}
\hline & Plant & Control & RS & $\begin{array}{l}\text { ADS } \\
\mathbf{4 8 d}\end{array}$ & $\begin{array}{l}\text { ACS } \\
\mathbf{2 d}\end{array}$ & $\begin{array}{l}\text { ACS } \\
\mathbf{5 d}\end{array}$ & $\begin{array}{l}\text { ACS } \\
\mathbf{1 0 d}\end{array}$ & $\begin{array}{l}\text { ACS } \\
\mathbf{1 4 d}\end{array}$ & $\begin{array}{l}\text { ACS } \\
\mathbf{6 0 d}\end{array}$ \\
\hline Germi & $\mathrm{SF}$ & $70 \pm 0$ & $30 \pm 5$ & $0 \pm 0$ & $45 \pm 0$ & $75 \pm 4$ & $80 \pm 11$ & $75 \pm 4$ & $75 \pm 0$ \\
nation & $\mathrm{CF}$ & $95 \pm 4$ & $85 \pm 0$ & $70 \pm 4$ & $25 \pm 14$ & $20 \pm 11$ & $20 \pm 4$ & $85 \pm 8$ & $90 \pm 4$ \\
$(\boldsymbol{\%})$ & $\mathrm{MG}$ & $100 \pm 0$ & $60 \pm 0$ & $0 \pm 0$ & $60 \pm 8$ & $45 \pm 11$ & $45 \pm 0$ & $60 \pm 0$ & $60 \pm 8$ \\
Shoot & $\mathrm{SF}$ & $103.9 \pm 32.2$ & $30.0 \pm 13.8$ & $0.0 \pm 0.0$ & $51.7 \pm 30.4$ & $49.3 \pm 38.2$ & $75.3 \pm 25.1$ & $97.7 \pm 28.7$ & $70.3 \pm 32.2$ \\
Lengh & $\mathrm{CF}$ & $64.7 \pm 19.2$ & $42.2 \pm 9.9$ & $28.0 \pm 22.2$ & $43.3 \pm 17.0$ & $35.8 \pm 25.4$ & $45.0 \pm 19.2$ & $60.6 \pm 14.4$ & $71.1 \pm 19.8$ \\
$(\mathbf{m m})$ & $\mathrm{MG}$ & $77.3 \pm 14.3$ & $44.3 \pm 21.2$ & $0.0 \pm 0.0$ & $12.3 \pm 7.2$ & $9.4 \pm 3.7$ & $9.3 \pm 4.9$ & $25.0 \pm 16.1$ & $29.6 \pm 20.6$ \\
\hline
\end{tabular}

The correlation between the germination and humification indexes . The parabolic curves fitted $(R \geqslant 0.9904)$ the correlations of DP, PHA and GI in three plants (Fig.4). In Fig.4 the negative slop of SF were found and it's predicted to occur in MG, agreed with the decreasing trend in subchronic tests from ACS 14d to 60d. Furthermore, it was confirmed in a 6-month compost [14], which observed GI of co-composting and found it got the highest at the second or third month then decreased in last months. It's worthy to pay attention to this trend. Although the report explained this by the action of 
phytotoxic substances and high ionic charge of water-soluble extracts, it was not evident in this study (Table 1). Instead of pollutant burdens, incomplete stabilization with the release of ammonium influenced the phytotoxicity mainly $[7,14,15]$ accorded with the decrease of TEN. As humification was one of the important factors of plant growth, it was reported the mix of humic acid (HA) and fulvic acid (FA) increased maize growth and yield [16]. So it implied small value of DP and PHA at first reflected the degradation of non-persistent pollutants and ammonia nitrogen release [18] caused low value of GI. Recently, it was found FA increased shoot growth of wheat through inhibiting uptake and translocation of $\mathrm{Cr}$ and increased plant biomass, photosynthetic pigments, and antioxidant enzymes while reduced metal concentration [17]. Consequently the lack of FA mainly caused that DP was up to 1.272 and explained the negative slop and plant growth decrease with 60-day compost.

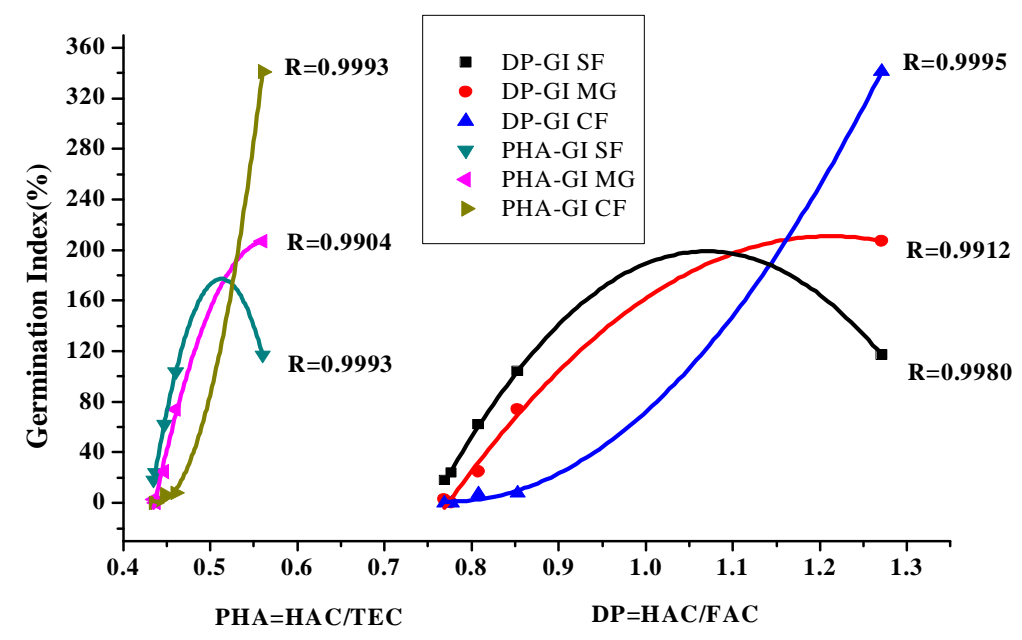

Fig.4. The parabolic correlation between DP, PHA and GI of 3 seed species and 5 sludges.

\section{Conclusions}

The phytotoxicity of sewage sludge tends to increase in high-rate anaerobic digestion but decrease in aerobic composting. As the composting time increased, the stability and phytotoxicity reduction of sludge increased gradually and reached the highest value at the 14th day and then decreased. The fresh, dry biomass and shoot length of ADS 60d were not better than ADS 14d.

The parabolic correlation of humification and phytotoxicity showed the minimal GI happened with the low value of DP and PHA implied the incomplete stabilization and humification might be the main factors of phytotoxicity in ADS. At the late period of aerobic composting, the lack of FA possibly caused the higher humification index. The finding points out that after high-rate anaerobic digestion, aerobic composting is a necessary and effective way to reduce sludge phytotoxicity before land application, but the composting time is not the longer the better.

\section{Acknowledgements}

The work was financially supported by the National Natural Scientific Foundation of China (51408423), National Water Pollution Control and Management Technology Major Projects (2013ZX07315003), National Key Technology Support Program (2014BAC29B01) and Postdoctoral Science Foundation of China (2015T80452).

\section{References}

[1] Information on http://www.chyxx.com/industry/201604/410147.html 
[2] J.A. Alburquerque, C. de la Fuente, A. Ferrer-Costa, L. Carrasco, J. Cegarra, M. Abad, and M.P. Bernal: submitted to Biomass and Bioenergy (2012)

[3] G. Mininni, A.R. Blanch, F. Lucena, and S. Berselli: submitted to Environmental Science and Pollution Research International (2015)

[4] K.M. Yang, C.Y. Zhang, Y. Zhang, G.S. Shun: submitted to China Resource Comprehensive Utilization (2012)

[5] M. Himanen, K. Hanninen: submitted to Bioresource Technology (2011)

[6] V. Tigini, M. Franchino, F. Bona, and G.C. Varese: submitted to The Science of The Total Environment (2016)

[7] M.E. Silva, L.T. Lemos, M.M. Bastos, O.C. Nunes, and A.C. Cunha-Queda: submitted to Bioresource Technology (2013)

[8] X. Domene, W. Ramirez, S. Mattana, J.M. Alcaniz, and P. Andres: submitted to Environmental Pollution (2008)

[9] G. Visioli, F.D. Conti, C. Gardi, and C. Menta: submitted to Bulletin of Environmental and Toxicology (2014)

[10] A. Branazini, and M.S. Zubillage: submitted to International Journal of Phytoremediation (2010)

[11] G. Visioli, C. Menta, C. Gardi, and F.D. Conti: submitted to Chemosphere (2013)

[12] A. Fuentes, M. Llorens, J. Saez, M.I. Aguilar, A.B. Perez-Marin, J.F. Orturio, and V.F. Meseguer: submitted to Environmental Pollution (2006)

[13] G. Masciandaro, C. Macci, S. Doni and B. Ceccanti: submitted to Journal of The Science of Food and Agriculture (2010)

[14] L. El Fels, M. Zamama, A. El Asli, and M. Hafidi: submitted to International Biodeterioration \& Biodegradation (2014)

[15] W.A. Ramirez, X. Domene, O. Ortiz, and J.M. Alcaniz: submitted to Bioresource Technology (2008)

[16] K. Matysiak, S. Kaczmarek, and R. Krawczyk: submitted to Acta Sci Pol Agric (2011)

[17] S. Ali, S.A. Bharwana, M. Rizwan, M. Farid, S. Kanwal, Q. Ali, M. Ibrahim, R.A. Gill, and M.D. Khan: submitted to Environmental Science and Pollution Research International (2015)

[18] W.A. Ramirez, X. Domene, P. Andres, and J.M. Alcaniz: submitted to Ecotoxicology (2008) 